\title{
1 SARS-CoV-2 PLpro whole human proteome cleavage prediction and
}

2 enrichment/depletion analysis

Lucas Prescott ${ }^{1 *}$

${ }^{1}$ Independent Researcher, Boston, Massachusetts, United States of America

6

$7 \quad *$ Corresponding author

8 E-mail: Iskywalker2015@gmail.com

9

10 Keywords: SARS-CoV-2, COVID-19, coronavirus, protease, deubiquitinase, proteomics, PLpro, machine 11 learning, neural networks 


\section{Abstract}

A novel coronavirus (SARS-CoV-2) has caused a pandemic that has killed millions of people, worldwide vaccination and herd immunity are still far away, and few therapeutics are approved by regulatory agencies for widespread use. The coronavirus 3-chymotrypsin-like protease (3CLpro) is a commonly investigated target in COVID-19, however less work has been directed toward the equally important papain-like protease (PLPro). PLpro is less characterized due to its fewer and more diverse cleavages in coronavirus proteomes and the assumption that it mainly modulates host pathways with its deubiquitinating activity. Here, I extend my previous work on 3CLpro human cleavage prediction and enrichment/depletion analysis to PLpro.[1] Using three sets of neural networks trained on different taxonomic ranks of dataset with a maximum of 463 different putative PLpro cleavages, Matthews correlation coefficients of $0.900,0.948$, and 0.966 were achieved for Coronaviridae, Betacoronavirus, and Sarbecovirus, respectively. I predict that more than 1,000 human proteins may be cleaved by PLpro depending on diversity of the training dataset and that many of these proteins are distinct from those previously predicted to be cleaved by 3 CLpro. PLpro cleavages are similarly nonrandomly distributed and result in many annotations shared with 3 CLpro cleavages including ubiquitination, poly(A) tail and $5^{\prime}$ cap RNA binding proteins, helicases, and endogenous viral proteins. Combining PLpro with 3CLpro cleavage predictions, additional novel enrichment analysis was performed on known substrates of cleaved E3 ubiquitin ligases with results indicating that many pathways including viral RNA sensing are affected indirectly by E3 ligase cleavage independent of traditional PLpro deubiquitinating activity. As with 3CLpro, PLpro whole proteome cleavage prediction revealed many novel potential therapeutic targets against coronaviruses, although experimental verification is similarly required.

\section{Introduction}

Coronavirus genomes contain multiple open reading frames, the largest of which contains two nonstructural cysteine proteases, nsp3/PLpro and nsp5/3CLpro, which cleave its polyprotein into functional proteins. 3 CLpro cleaves 11 conserved sights in all coronavirus proteomes, and PLpro cleaves only 2 or 3 sites depending on whether nsp 1 is functional. In addition to many other multifunctional domains, PLpro contains two papain-like domains with different properties, the first of which is sometimes inactive.[2]

Most experimental work on PLpro focuses on its deubiquitinating and delSGylating activity[3] and faster kinetics relative to the human homologs USP7 and USP14.[4] Proteins and pathways disrupted by this activity include retinoic acid-inducible gene I (RIG-I), melanoma differentiationassociated protein 5 (MDA5), TNFR-associated factors (TRAF3/6), stimulator of interferon genes (STING), TANK-binding kinase 1 (TBK1), nuclear factor $\mathrm{k}$-light-chain-enhancer of activated B cells (NF-KB), interferon regulatory factor 3 (IRF3), type-I interferon production, Janus kinases and signal transducers and activators of transcription (JAK-STAT), and collagen expression via transforming growth factor (TGFB1).[5-16] Separately, simple sequence alignments of the three SARS-CoV-2 cleavages with human proteins ranked by percent identity led Reynolds et al. to propose a few dozen human proteins to be directly cleaved by PLpro.[17] This method does not account for position-dependent residue importance or any physiochemical trends and therefore likely resulted in many false positives, yet they were able to demonstrate in vitro cleavage of protein $\mathrm{S}, \mathrm{MHC}-\alpha / \beta$ isoforms (MYH6/7), forkhead box P3 (FOXP3), and epidermal growth factor receptor (ERBB4). IRF3,[18] Unc-51 like autophagy activating kinase (ULK1),[19] proto-oncogene SRC, Kelch domain-containing protein 10 (KLHDC10), and bone marrow stromal antigen 1 (BST1)[20] were also independently demonstrated to be cleaved by PLpro in vitro.

The high number of known 3CLpro cleavages has allowed for sequence logos and machine learning techniques to be applied to coronavirus and human sequences.[1, 21] PLpro, however, is less characterized because (1) fewer cleavage sites are known, (2) these cleavages are not as conserved as $3 C$ pro cleavages, and it is unknown whether (3) any alpha- or betacoronaviruses are missing a cleavage 
before nsp2, (4) all gamma- and deltacoronaviruses are missing a cleavage before nsp2, or (5) whether the many uncharacterized coronaviruses have one or two functional papain-like domains and what differences they may have.

\section{Methods}

\section{Data Set Preparation}

A complete, manually reviewed human proteome containing 20,350 sequences (not including alternative isoforms) was retrieved from UniProt/Swiss-Prot (proteome:up000005640 AND reviewed:yes).[22]

Additional coronavirus polyprotein cleavages were collected from GenBank. [23] Searching for all combinations of "orf/pp" and "1/1a/1ab" within the family Coronaviridae returned 12,770 different, complete polyproteins with 463 different cleavages manually discovered using the Clustal Omega multiple sequence alignment server.[24-26] Unlike 3CLpro, no PLpro domain is found in the monotypic Microhyla letovirus 1 in Coronaviridae. Similar domains are characterized in Arteriviridae and in some of Tobaniviridae, although none of these sequences were aligned here.[27] All unbalanced positive cleavages were used for subsequent classifier training in addition to all other 33,185 uncleaved coronavirus sequence windows with at least two glycines or alanines in the $\mathrm{P} 2, \mathrm{P} 1$, or $\mathrm{P} 1$ ' positions, totaling 33,648 samples.

\section{Cleavage Prediction}

As in my previous work on 3CLpro,[1] sequence logo-based logistic regression and naïve Bayes classification and physiochemical and one-hot encoded neural networks (NNs) were used for prediction of cleavage sites.[28]

\section{Enrichment Analysis}

Protein annotation, classification, and enrichment analysis was performed using the Database for Annotation, Visualization, and Integrated Discovery (DAVID) 6.8.[29, 30] Tissue (UP_TISSUE and UNIGENE_EST_QUARTILE), InterPro, direct Gene Ontology (GO includes cellular compartment (CC), biological process (BP), and molecular function (MF)), Reactome pathways, sequence features, and keywords annotations were all explored, and only annotations with Benjamini-Hochberg-corrected pvalues less than 0.05 were considered statistically significant. Cleaved E3 ubiquitin ligases were matched to their respective substrates for additional enrichment analysis using the UbiNet 2.0 database.[31] All training data, prediction methods, and results can be found on GitHub (https://github.com/Luke8472NN/NetProtease).

\section{Results}

Due to the greater difficulty aligning putative cleavages and more variable domains of $n s p 3$, the assumption that SARS-CoV-2 PLpro can cleave all aligned sites in divergent coronaviruses is less plausible than for $3 C$ Lpro. First, some entries in this dataset containing one, two, or four glycines or alanines may be incorrectly shifted by one or two residues because they are difficult to align with PLpro's consensus $[\mathrm{KL}] \mathrm{K}[\mathrm{GAR}][\mathrm{GA}]^{\wedge}[\mathrm{GA}]$. The $\mathrm{P} 1^{\prime}$ glycine or alanine is also not applicable when cleaving isopeptide bonds for deubiquitination, however lysine's bound side chain somewhat resembles another small, hydrophobic peptide bond. Glycine is the smallest and most similar amino acid, and alanine is the second smallest, also resembling the hydrophobicity of lysine's side chain (Figure 1) and potentially similarly protecting the oxyanion of the tetrahedral intermediate from water in the tryptophancontaining oxyanion hole.[6] If this is correct, then ubiquitin's arginine residue should be in the P3 position and not the $\mathrm{P} 2$ position as hinted by many sequences aligned here, particularly nsp $1 / 2$ cleavages (Figure 2). Molecular docking or crystallography are, however, required to prove these chemical similarities relevant. Additionally complicating the problem, dimensionality reduction of 
putative cleavages did not cluster by order as well as for 3CLpro, and nsp1/2 cleavages missing from all gamma- and deltacoronaviruses have a distinct sequence logo (Figure 2).
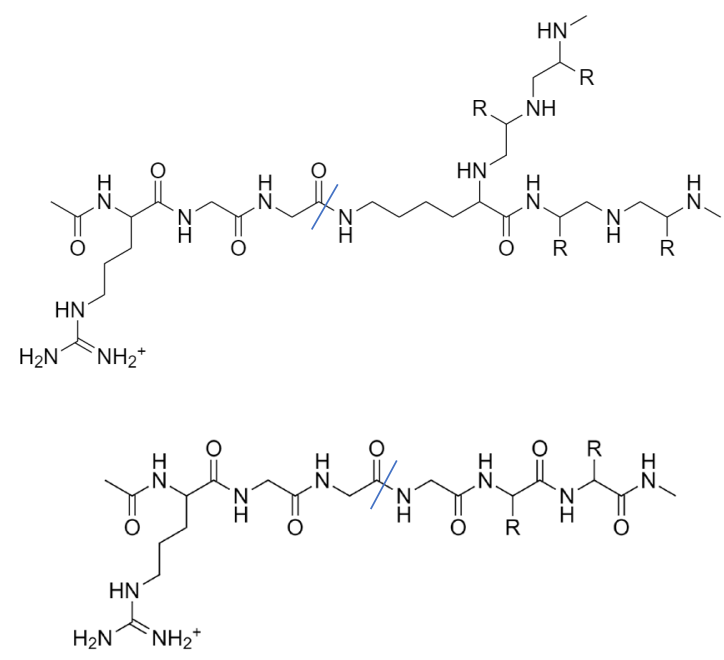

Figure 1: Chemical similarity between (A) ubiquitin-target $R G G^{\wedge} \mathrm{K}$ isopeptide bond and (B) $R G G^{\wedge} G$ peptide bond.
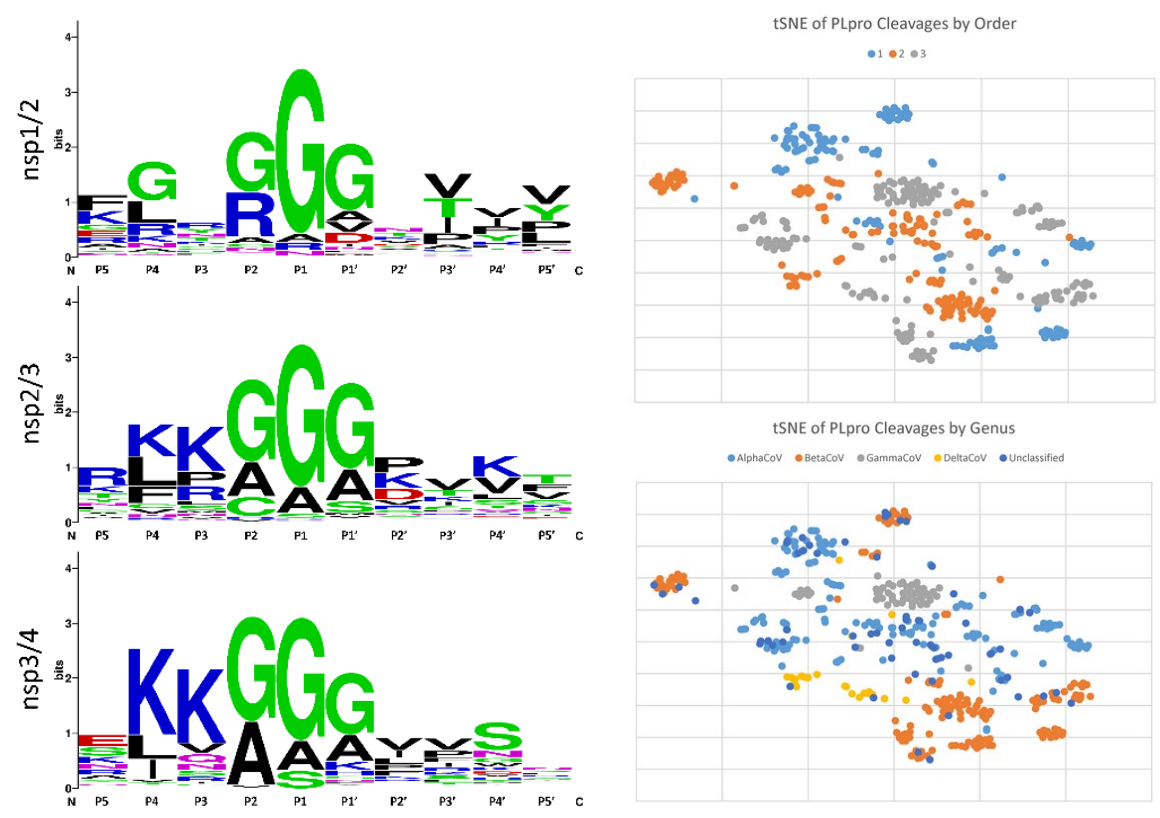

Figure 2: (A) Sequence logos for the three cleavage sites in coronaviruses[32] and (B) one-hot encoded tSNE colored by order and (C) by genus.[33]

With no easy way to prove functionality or activity of divergent papain-like domains and therefore the suitability of distant cleavages to be included into a single, unweighted dataset, machine learning models were trained on three different taxonomic ranks of data (all Coronaviridae, only Betacoronavirus, and only Sarbecovirus). Predicting sequence-dependent kinetic rates would be even more difficult than this classification given that swine acute diarrhea syndrome coronavirus (SADS-CoV) PLpro catalytic efficiency varies by two orders of magnitude depending of substrate[34] and also given that SARS-CoV-1 and -2 PLpro share $83 \%$ identity yet are known to preferentially cleave ubiquitin vs ISG15, respectively.[35] Preferences for isopeptidase over peptidase activity,[36] polyubiquitin chains 
over ISG15 or monoubiquitin,[37] and K48 over K63 ubiquitin linkages[38] also vary between viral lineages.[39, 40] The distantly related foot-and-mouth disease virus (FMDV) leader protease even has irreversible delSGylating activity by leaving ISG15's C-terminal diglycine on its target lysine.[41] It is fair to assume, however, that cleavages predicted by all three sets of models can occur in many infections no matter viral lineage. The size and imbalances of these final datasets are listed in Table 1 . The greater diversity in the higher taxonomic datasets caused many more random sequences and human proteins to be predicted to be cleaved, however enrichment/depletion analysis proved robust against this.

Table 1: Size and imbalance of the three datasets used to train machine learning models and the results of the final NN.

\begin{tabular}{|l|l|l|l|}
\hline Rank & Coronaviridae & Betacoronavirus & Sarbecovirus \\
\hline nsp1/2 cleavages & 133 & 49 & 16 \\
\hline nsp2/3 cleavages & 153 & 60 & 23 \\
\hline nsp3/4 cleavages & 177 & 46 & 13 \\
\hline Total cleaved & 463 & 155 & 52 \\
\hline Total uncleaved & 33185 & 10013 & 2742 \\
\hline$\%$ Cleaved & $1.38 \%$ & $1.52 \%$ & $1.86 \%$ \\
\hline Single network 20\% test MCC & 0.895 & 0.934 & 0.988 \\
\hline Ensemble 20\% test MCC & 0.900 & 0.948 & 0.966 \\
\hline $\begin{array}{l}\text { Random cleavage frequency } \\
\text { between 3 Gs or As }\end{array}$ & $2.00 \%$ & $0.54 \%$ & $0.07 \%$ \\
\hline $\begin{array}{l}\text { Random cleavage frequency } \\
\text { between } \geq 2 \text { Gs or As }\end{array}$ & $0.38 \%$ & $0.23 \%$ & $0.05 \%$ \\
\hline Random cleavage frequency & $0.019 \%$ & $0.012 \%$ & $0.003 \%$ \\
\hline $\begin{array}{l}\text { Average residues between } \\
\text { random cleavages }\end{array}$ & 5300 & 8300 & 33000 \\
\hline Human proteins cleaved & 1381 & 980 & 154 \\
\hline
\end{tabular}

As with 3CLpro predictions, NNs outperformed all other classifiers no matter taxonomic rank (Figure 3). This performance difference was most noticeable for the whole Coronaviridae dataset where single sequence logos cannot learn its multiple populations. Interestingly, physiochemical encoding also proved to describe sequences less efficiently than one-hot encoding and sequence logos for the Sarbecovirus-only dataset. The optimized hyperparameters for NNs with one-hot encoding were Adam solver, rectifier (ReLU) activation, 0.000001 regularization, no oversampling, and 1 hidden layer with 100 neurons. Combining networks into ensembles again improved accuracy and stability, so the final results were generated with triplicate 10 -fold cross-validated networks with average $20 \%$ test Matthews correlation coefficients (MCC) of 0.900, 0.948, and 0.966 for Coronaviridae, Betacoronavirus, and Sarbecovirus, respectively. These accuracies, frequency cleaving random amino acid sequences, and number of human proteins with predicted cleavages are also listed in Table 1. 
bioRxiv preprint doi: https://doi. org/10.1101/2021.10.04 462902; this version posted October 6, 2021. The copyright holder for this preprint (which was not certified by peer review) is the author/funder, who has granted bioRxiv a license to display the preprint in perpetuity. It is made available under aCC-BY 4.0 International license.

Coronaviridae Dataset Train/Test Split Fraction vs Test MCC

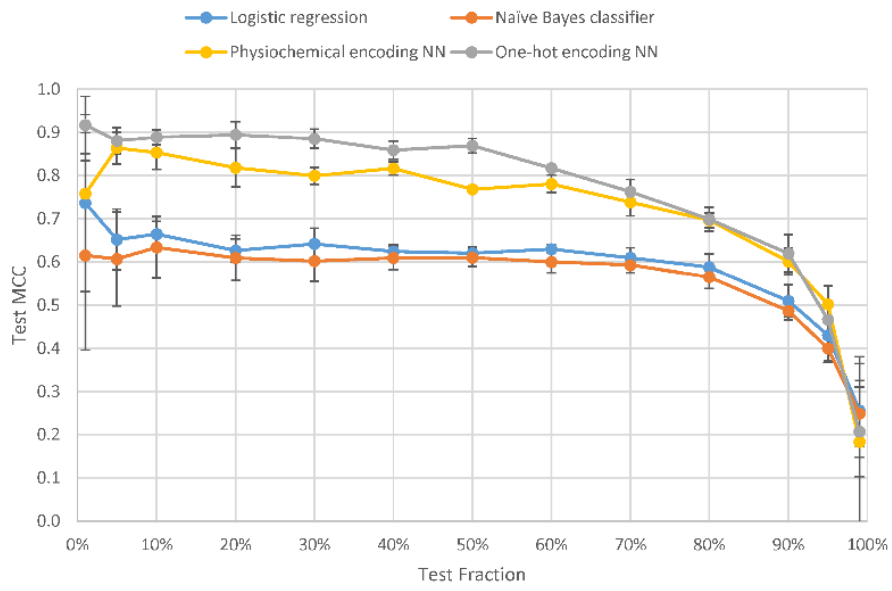

Betacoronavirus Dataset Train/Test Split Fraction vs Test MCC

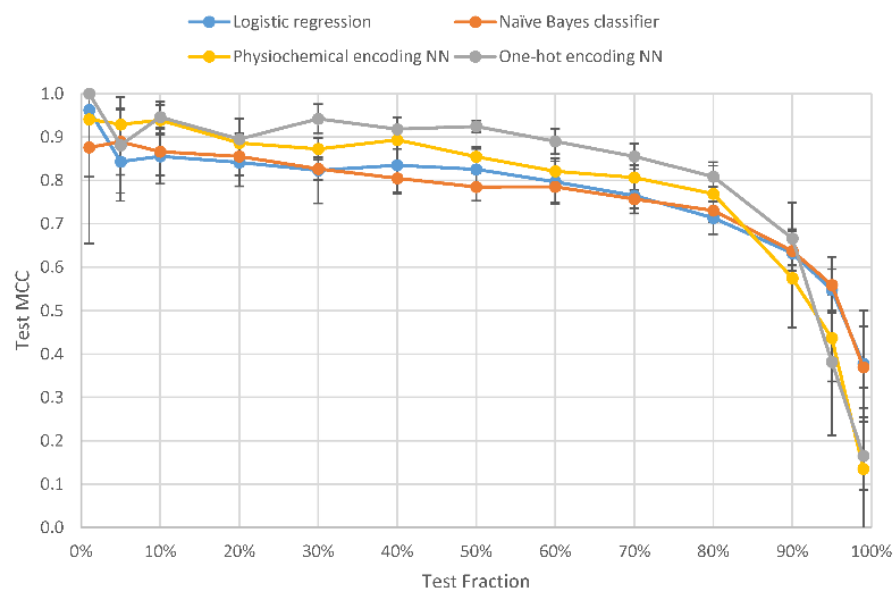

152

Sarbecovirus Dataset Train/Test Split Fraction vs Test MCC

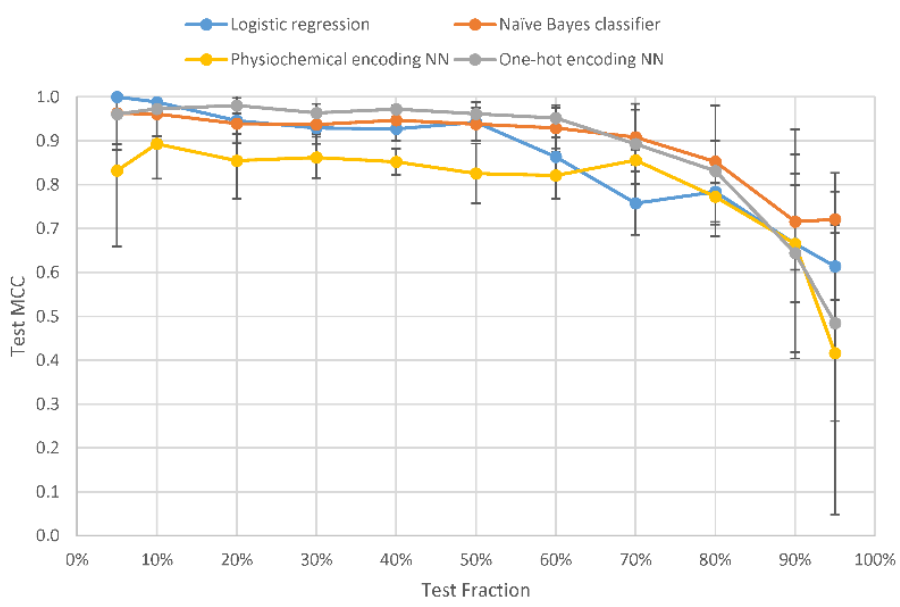

153

154

155

156
Figure 3: Train/test split fraction vs MCC demonstrating that performance quickly approaches a limit for all classifiers no matter taxonomic rank ((A) Coronaviridae, (B) Betacoronavirus, (C) Sarbecovirus). 


\section{Discussion}

Enrichments and depletions were surprisingly shared between NN replicates, resampled training data, and between the three taxonomic ranks of training data, although the Sarbecovirus-specific list returned fewer statistically significant annotations due to its smaller size. As expected from SARS-CoV-2 pathogenesis and from previous 3CLpro results, [1] epithelium (tongue, larynx, pharynx, trachea) and brain were enriched, and olfactory receptors were depleted. As expected from the sequence logo, glycine- and alanine-rich proteins, such as collagens, were also enriched. Cytoskeletal and motor proteins were enriched as with 3CLpro due to their longer lengths, although their cleavage is likely still biologically relevant. In fact, this length bias is even larger in Reynolds et al.'s sequence identity method,[17] and surprisingly few of their predictions were shared in this PLpro prediction dataset, namely protection of telomeres 1 (POT1), membrane-associated guanylate kinase inverted 2 (MAGI2), multiple EGF-like domains 10 (MEGF10), myocardin, inactive RNASE10, amyloid beta A4 precursor protein-binding (APBB2), and myomesin-1. FOXP3 was also the only experimentally validated protein to be successfully predicted here.[18-20] This indicates that, as with 3CLpro prediction, any inaccuracies in prediction methods are amplified when applied to the entire human and must be experimentally verified, however enrichment analyses are robust against this variability.[1]

The ubiquitination pathway was expectedly enriched given one of PLpro's main functions is to deubiquitinate host proteins. Global effects of PLpro's preference for particular ubiquitin chains or branchings are poorly characterized, and no peptide bond cleavages within E1, E2, or E3 enzymes are known. Combining these PLpro predictions with my previous 3CLpro dataset,[1] global effects on these pathways become clearer. Of the ten E1 enzymes,[42] two are cleaved by 3CLpro: UBA2 and UBA5. UBA2 is half of the only heterodimer required for SUMOylation and is cleaved in its C-terminal ubiquitinlike domain required for transfer to the only SUMO E2 conjugating protein, UBC9,[43] which is not cleaved by either protease. Interestingly, coronavirus nucleocapsid proteins are intentionally SUMOylated to promote their homo-oligomerization, interfere with cell division, and/or localize to the nucleus or nucleolus to alter viral vs host transcription or ribosomal biogenesis. [44-46] One downstream consequence of disrupted SUMOylation is reduced stability of STING,[47] which is already disrupted by PLpro deubiquitination.[48, 49] TRIM38 (SUMOylating) is not, but SENP2 (deSUMOylating) is cleaved, indicating that STING regulation probably differs between early and late phase infection. PLpro's additional deubiquitinating activity or cleavage of related enzymes may also change the period of SUMOylation and ubiquitination oscillations, impacting global proteasomal degradation kinetics.[50] Lastly, depletion of E2-conjugated SUMO stores may lead to preferential ubiquitination and degradation of p53[51] by RCHY1 later in infection,[36] which is already directly enhanced by PLpro SARS-unique domain, [52] and $\mathrm{IKB} \alpha$,[53] which activates NF-KB and worsens SARS-related mortality in mice.[54] UBA5, however, is the only UFMylation-specific E1 enzyme with implications in reticulophagy and hematopoiesis, and its only E2 conjugating protein, UFC1, is not cleaved by either protease.[55] First, UFMylation of DDRGK1, a reticulophagy protein activated by ribosome stalling,[56] may play a role in encouraging ribosomal frameshifts by forming double-membrane vesicles (DMVs) utilized by coronaviruses for replication[57] or by compartmentalizing ribosomes with viral RNA inside DMVs as with influenza A virus. [58] Second, like SUMOylation, UFMylation of p53 antagonizes ubiquitination and subsequent degradation.[59] Lastly, UFM1 depletion may encourage macrophage responses to interferon gamma,[60] contributing to the positive feedback with T cells in COVID-19-associated alveolar inflammation.[61] Although none of the few ISG15 enzymes are cleaved by either coronavirus protease, NEDD4, which is likely eventually inhibited by free ISG15 released by PLpro's delSGylating activity, [62] is predicted to be cleaved by 3 CLpro[1] and has been suggested as a therapeutic target for COVID-19 due to its upregulation in infected cells and probable involvement in egress via non-degradative ubiquitination of viral proteins.[63] The counterintuitive proviral activity of NEDD4 yet potential 
inhibition directly by $3 C$ Lpro and indirectly by PLpro hints that its regulation may be infection time dependent.

Interpreting viral intent behind E2 and E3 enzyme cleavages is much more difficult, however similar enrichment analysis can be performed on the substrates specific to cleaved E3 ligases. Combining all predicted cleavages from the three PLpro datasets and the one 3CLpro dataset[1] and applying to UbiNet 2.0 E3-substrate interactions,[31] 38\% of substrates were directly cleaved by the viral proteases, and $47 \%$ of substrates had E3 ligases cleaved by viral proteases. Because most ubiquitination tags substrates for proteasomal degradation, E3 ligase cleavage would effectively upregulate affected pathways. Although $19 \%$ of these cleavages were overlapping and therefore had unknown up- vs downregulation, most noteworthy enrichments were preserved after removal of this overlap from the list of substrates with cleaved E3 ligases. Noteworthy affected pathways include RAP1 related to cell adhesion and junction formation, PI3K/AKT related to cell survival and proliferation, p53 related to apoptosis, clathrin-dependent and -independent endocytosis, $\mathrm{Fc} \gamma$ and Fce receptor signaling, and viral RNA-sensing RIG-I and TLR3 signaling to IRF3/7 and NF-KB and downstream interferon-stimulated STAT1/3. Given these pathways, particularly viral RNA sensing, are affected more directly by many other viral proteins early in infection, pathway activation via cleavage of E3 ligases independent of direct PLpro deubiquitination followed by reduced substrate turnover rate is likely only relevant later in infection once responses to interferon have begun.

In addition to directly deubiquitinating host proteins, PLpro cleavage of lysyl oxidases (LOXL2/3) may disrupt the balance of lysine/acetyllysine/allysine on histones or non-histone proteins [64] to prevent crosslinking or monoubiquitination or disrupt acetylation of ubiquitin itself to prevent polyubiquitination. [65] For example, preventing LOXL3 deacetylation of STAT3 or relatedly cleaving chromodomain-helicase-DNA-binding protein 8 (CHD8), a STAT3 transcriptional repressor, could be part of how SARS-CoV-2 modulates immune cell differentiation[66] or the STAT1/STAT3 ratio.[67]

Outside ubiquitination, RNA binding also stood out as a relevant enrichment because many antiviral pathways attempt to directly degrade viral RNA. Interestingly, the RGG/RG motif present in many RNA-binding proteins[68] is likely enriched in cleavages due to their similarity to PLpro's sequence logo. Noteworthy cleaved proteins with RNA binding ability include poly(A) tail-associated Ras GTPaseactivating protein-binding protein 2 (G3BP2), cleavage and polyadenylation specificity factors (CPSF5/7), zinc finger $\mathrm{C} 3 \mathrm{H} 1$ domain-containing protein $(\mathrm{ZFC} 3 \mathrm{H} 1)$, and zinc finger $\mathrm{CCHC}$ domain-containing protein 14 (ZCCHC14) and 5' cap-associated interferon-induced protein with tetratricopeptide repeats 2 (IFIT2) and dual specificity phosphatase 11 (DUSP11). G3BP2 is involved in stress granule formation and documented to be inhibited by SARS-CoV-2 nucleocapsid protein.[69, 70] Cleavage of RNA cleavage factor Im subunits CPSF5/7 likely contributes to enhanced viral RNA translation by trapping host premRNA in the nucleus as with influenza virus.[71] ZFC3H1 is a subunit of the poly(A) tail exosome targeting (PAXT) complex which targets longer and more polyadenylated RNAs than the traditional nuclear exosome targeting (NEXT) complex[72] with subunit ZCCHC14 also cleaved by PLpro and others cleaved by 3 CLpro.[1] In addition to variable length polyadenylation over infection and between whole genomes and subgenomic mRNAs, $[73,74]$ time-dependent antagonism of these two complexes by PLpro and 3CLpro may be another method coronaviruses control their relative rates of replication vs translation, the ratios of their subgenomic mRNAs, or the ratio of orf1a to orf1ab and resulting proteins. Given hepatitis B virus (HBV) and human cytomegalovirus (HCMV) exploit ZCCHC14 to elongate their poly(A) tails with errors to enhance stability against CCR4-NOT, coronaviruses should also be experimentally surveyed for similar not-so-pure poly(A) tails.[75] In fact, CCR4-NOT subunits 1 and 4, the latter with E3 ubiquitin ligase activity, are cleaved by 3CLpro.[1] In the cytoplasm, this cleavage probably stabilizes viral RNA and viral translation, but disrupting E3 ligase activity in the nucleus would stabilize its target JARID1C, a histone H3 K4 demethylase, globally repressing host transcription so that ribosomes can be directed more toward viral transcripts.[76] As for the two 5' cap-associated proteins, interferon- 
stimulated IFIT2 inhibits eukaryotic initiation factor 3 (elF3)-mediated translation of viral RNAs without cap 2'-O-ribose methylation, a pathway coronaviruses are sensitized to by inhibiting nsp16's 2'-O-ribose methyltransferase activity, $[77,78]$ and DUSP11 with $5^{\prime}$-triphosphatase and diphosphatase activity can either directly sensitize viral RNA to exoribonucleases as with hepatitis C virus (HCV)[79] or control RIG-I responses by altering viral or host triphosphate RNA balances.[80, 81] RNA helicases are relatedly enriched yet as with 3 CLpro cleavages have varying proviral and antiviral effects. Novel PLpro cleavages include neuron navigator 2 , which possesses both helicase and exoribonuclease activity and is expressed in sensory nervous tissue susceptible to coronavirus infection, and DEAD box protein DDX42, which was recently shown to have broad antiviral activity including SARS-CoV-2 inhibition.[82]

Lastly, as with 3CLpro,[1] endogenous retroviral proteins were enriched in cleavages, yet HERV-K genes were recently found to be more expressed in tracheal aspirates of patients with severe COVID-19 compared to uninfected and mildly infected patients. [83] Because this expression correlates with mortality, it is worth investigating inhibiting this mechanism with antiretroviral drugs commonly used against HIV.[84] HIV protease inhibitors cannot directly inhibit coronavirus proteases at reasonable concentrations due to their difference in mechanism,[85] and lopinavir-ritonavir did not improve outcomes of patients hospitalized with COVID-19,[86] indicating that targeting HERVs in COVID-19 may be difficult or too indirect for therapeutic efficacy.

\section{Conclusion}

Many results from my previous work on 3CLpro cleavages including enrichment of epithelium and brain, depletion of olfactory receptors, and enrichment of ubiquitination, viral RNA stabilizing and degrading mechanisms, and endogenous viral proteins are preserved in PLpro predictions.[1] Combination of the two cleavage datasets allowed for additional enrichment analysis of known substrates of cleaved E3 ubiquitin ligases, revealing that coronaviruses can regulate ubiquitination in host pathways independent of direct PLpro deubiquitinating activity. Experimental verification of these cleavages and characterization of the time dependence of these ubiquitination effects may reveal novel drug targets against COVID-19 or future human coronaviruses. Lastly, expansion of the training dataset to more divergent PLpro-containing Nidoviruses may be helpful in developing methods to phylogenetically weight aligned cleavage datasets to maximize accuracy for viral lineages or hosts of interest.

\section{Acknowledgements}

I am very grateful for my mother, Victoria Prescott, Esq., and friends who have given me invaluable help and advice throughout my work on this project.

\section{References}

1. Prescott L. SARS-CoV-2 3CLpro whole human proteome cleavage prediction and enrichment/depletion analysis. bioRxiv [Preprint]. 2020 Aug. doi: 10.1101/2020.08.24.265645

2. Lei J, Kusov Y, Hilgenfeld R. Nsp3 of coronaviruses: Structures and functions of a large multi-domain protein. Antiviral Res. 2018 Jan;149:58-74. doi: 10.1016/j.antiviral.2017.11.001 PMID: 29128390

3. Neimeyer D, Mosbauer K, Klein EM, Sieberg A, Mettelman RC, Mielech AM, et al. The papain-like protease determines a virulence trait that varies among members of the SARS-coronavirus species. PLOS Pathog. 2018 Sep;14(9):e1007296. doi: 10.1371/journal.ppat.1007296 PMID: 30248143

4. Ratia K, Saikatendu KS, Santarsiero BD, Barretto N, Baker SC, Stevens RC, et al. Severe acute respiratory syndrome coronavirus papain-like protease: structure of a viral deubiquitinating enzyme. PNAS. 2006 Apr;103(15):5717-22. doi: 10.1073/pnas.0510851103 PMID: 16581910

5. Masucci MG. Viral ubiquitin and ubiquitin-like deconjugases-Swiss Army knives for infection. Biomolecules. 2020 Aug;10(8):1337. doi: 10.3390/biom10081137 PMID: 32752270 
bioRxiv preprint doi: https://doi.org/10.1101/2021.10.04.462902; this version posted October 6, 2021. The copyright holder for this preprint (which was not certified by peer review) is the author/funder, who has granted bioRxiv a license to display the preprint in perpetuity. It is made available under aCC-BY 4.0 International license.

6. Baez-Santos YM, St John SE, Mesecar AD. The SARS-coronavirus papain-like protease: structure, function and inhibition by designed antiviral compounds. Antiviral Res. 2015 Mar;115:21-38. doi: 10.1016/j.antiviral.2014.12.015 PMID: 25554382

7. Frieman $M$, Ratia K, Johnston RE, Mesecar AD, Baric RS. Severe acute respiratory coronavirus papain-like protease ubiquitin-like domain and catalytic domain regulate antagonism of IRF3 and NF-KB signaling. J Virol. 2009 Jul;83(13):6689-705. doi: 10.1128/JVI.02220-08 PMID: 19369340

8. Barretto N, Jukneliene D, Ratia K, Chen Z, Mesecar AD, Baker SC. The papain-like protease of severe acute respiratory syndrome coronavirus has deubiquitinating activity. J Virol. 2005 Dec;79(24):15189-98. doi: 10.1128/JVI.79.24.15189-15198.2005 PMID: 16306590

9. Yang $X$, Chen X, Bian G, Tu J, Xing Y, Wang Y, et al. Proteolytic processing, deubiquitinase and interferon antagonist activities of Middle East respiratory syndrome coronavirus papain-like protease. J Gen Virol. 2014 Mar;95(3):614-26. doi: 10.1099/vir.0.059014-0 PMID: 24362959

10. Bailey-Elkin BA, Knaap RCM, Johnson GG, Dalebout TJ, Ninaber DK, van Kasteren PB, et al. Crystal structure of the Middle East respiratory syndrome coronavirus (MERS-CoV) papain-like protease bound to ubiquitin facilitates targeted disruption of deubiquitinating activity to demonstrate its role in innate immune suppression. J Biol Chem. 2014 Dec;289:34667-82. doi: 10.1074/jbc.M114.609644 PMID: 25320088

11. Li SW, Lai CC, Ping JF, Tsai FJ, Wan L, Lin YJ, et al. Severe acute respiratory syndrome coronavirus papain-like protease suppressed alpha interferon-induced responses through downregulation of extracellular signal-regulated kinase 1-mediated signalling pathways. J Gen Virol. 2011 May;92(5):1127-40. doi: 10.1099/vir.0.028936-0 PMID: 21270289

12. Xing $\mathrm{Y}$, Chen J, Tu J, Zhang B, Chen $\mathrm{X}$, Shi $\mathrm{H}$, et al. The papain-like protease of porcine epidemic diarrhea virus negatively regulates type I interferon pathway by acting as a viral deubiquitinase. J Gen Virol. 2013 Jul;94(7):1554-67. doi: 10.1099/vir.0.051169-0 PMID: 23596270

13. Matthews K, Schafer A, Pham A, Frieman M. The SARS coronavirus papain like protease can inhibit IRF3 at a post activation step that requires deubiquitination activity. Virol J. 2014 Dec;11:209. doi: 10.1186/s12985-014-0209-9 PMID: 25481026

14. Devaraj SG, Wang N, Chen Z, Chen Z, Tseng M, Barretto N, et al. Regulation of IRF-3-dependent innate immunity by the papain-like protease domain of the severe acute respiratory syndrome coronavirus. J Biol Chem. 2007 Nov;282:32208-21. doi: 10.1074/jbc.M704870200 PMID: 17761676

15. Wang CY, Lu CY, Li SW, Lai CC, Hua CH, Huang SH, et al. SARS coronavirus papain-like protease upregulates the collagen expression through non-Samd TGF- $\beta 1$ signaling. Virus Res. 2017 May;235:5866. doi: 10.1016/j.virusres.2017.04.008 PMID: 28414040

16. Liu G, Lee JH, Parker ZM, Acharya D, Chiang JJ, van Gent M, et al. ISG15-dependent activation of the sensor MDA5 is antagonized by the SARS-CoV-2 papain-like protease to evade host innate immunity. Nat Microbiol. 2021 Apr;6(4):467-78. doi: 10.1038/s41564-021-00884-1 PMID: 33727702

17. Reynolds ND, Aceves NM, Liu JL, Compton JR, Leary DH, Freitas BT, et al. The SARS-CoV-2 SSHHPS recognized by the papain-like protease. ACS Infect Dis. 2021 Jun;7(6):1483-502. doi: 10.1021/acsinfecdis.0c00866 PMID: 34019767

18. Moustaqil M, Ollivier E, Chiu HP, Van Tol S, Rudolffi-Soto $P$, Stevens $C$, et al. SARS-CoV-2 proteases PLpro and 3CLpro cleave IRF3 and critical modulators of inflammatory pathways (NLRP12 and TAB1): implications for disease presentation across species. Emerg Microbes Infect. 2021 Dec;10(1):178-95. doi: 10.1080/22221751.2020.1870414 PMID: 33372854

19. Mohamud Y, Xue YC, Liu H, Ng CS, Bahreyni A, Jan E, et al. The papain-like protease of coronaviruses cleaves ULK1 to disrupt host autophagy. Biochem Biophys Res Commun. 2021 Feb;540:75-82. doi: 10.1016/j.bbrc.2020.12.091 PMID: 33450483

20. Meyer B, Chiaravalli J, Gellenoncourt S, Brownridge P, Bryne DP, Daly LE, et al. Characterising proteolysis during SARS-CoV-2 infection identifies viral cleavage sites and cellular targets with 
therapeutic potential. Nat Commun. 2021 Sep;12(1):5553. doi: 10.1038/s41467-021-25796-w PMID: 34548480

21. Gao F, Ou HY, Chen LL, Zheng WZ, Zhang CT. Prediction of proteinase cleavage sites in polyproteins of coronaviruses and its applications in analyzing SARS-CoV genomes. FEBS Lett. 2003 Oct;553(3):451-6. doi: 10.1016/s0014-5793(03)01091-3 PMID: 14572668

22. The UniProt Consortium. UniProt: a worldwide hub of protein knowledge. Nucleic Acids Res. 2019 Jan;47(D1):D505-15. doi: 10.1093/nar/gky1049 PMID: 30395287

23. Benson DA, Cavanaugh M, Clark K, Karsch-Mizrachi I, Lipman DJ, Ostell J. GenBank. Nucleic Acids Res. 2017 Jan;45(D1):D37-42. doi: 10.1093/nar/gkw1070 PMID: 27899564

24. Sievers F, Wilm A, Dineen D, Gibson TJ, Karplus K, Li W, et al. Fast, scalable generation of highquality protein multiple sequence alignments using Clustal Omega. Mol Syst Biol. 2011 Oct;7:539. doi: 10.1038/msb.2011.75 PMID: 21988835

25. Goujon M, McWilliam H, Li W, Valentin F, Squizzato S, Paern J, et al. A new bioinformatics analysis tools framework at EMBL-EBI. Nucleic Acids Res. 2010 Jul;38(2):W695-9. doi: 10.1093/nar/gkq313 PMID: 20439314

26. McWilliam H, Li W, Uludag M, Squizzato S, Park YM, Buso N, et al. Analysis tool web services from the EMBL-EBI. Nucleic Acids Res. 2013 Jul;41(W1):W597-600. doi: 10.1093/nar/gkt376 PMID: 23671338

27. Bukhari K, Mulley G, Gulyaeva AA, Zhao L, Shu G, Jiang J, et al. Description and initial characterization of metatranscriptomic nidovirus-like genomes from the proposed new family Abyssoviridae, and from a sister group to the Coronavirinae, the proposed genus Alphaletovirus. Virology. 2018 Nov;524:160-71. doi: 10.1016/j.virol.2018.08.010 PMID: 30199753

28. Pedregosa F, Varoquaux G, Gramfort A, Michel V, Thirion B, Grisel O, et al. Scikit-learn: Machine Learning in Python. J Mach Learn Res. 2011 Oct;12:2825-30. http://jmlr.csail.mit.edu/papers/v12/pedregosa11a.html

29. Huang DW, Sherman BT, Lempicki RA. Systematic and integrative analysis of large gene lists using DAVID bioinformatics resources. Nat Protoc. 2009 Jan;4(1):44-57. doi: 10.1038/nprot.2008.211 PMID: 19131956

30. Huang DW, Sherman BT, Lempicki RA. Bioinformatics enrichment tools: Paths toward the comprehensive functional analysis of large gene lists. Nucleic Acids Res. 2009 Jan;37(1):1-13. doi: 10.1093/nar/gkn923 PMID: 19033363

31. Li Z, Chen S, Jhong JH, Pang Y, Huang KY, Li S, et al. UbiNet 2.0: a verified, classified, annotated and updated database of E3 ubiquitin-ligase-substrate interactions. Database (Oxford). 2021 Mar;2021:baab010. doi: 10.1093/database/baab010 PMID: 33693667

32. Crooks GE, Hon G, Chandonia JM, Brenner SE. WebLogo: A sequence logo generator. Genome Res. 2004 Jun;14:1188-90. doi: 10.1101/gr.849004 PMID: 15173120

33. van der Maaten L, Hinton G. Visualizing data using t-SNE. J Mach Learn Res. 2008 Nov;9:2579-605. doi: http://www.jmlr.org/papers/v9/vandermaaten08a.html

34. Wang L, Hu W, Fan C. Structural and biochemical characterization of SADS-CoV papain-like protease 2. Protein Sci. 2020 May;29(5):1228-41. doi: 10.1002/pro.3857 PMID: 32216114

35. Shin D, Mukherjee R, Grewe D, Bojkova D, Baek K, Bhattacharya A, et al. Papain-like protease regulates SARS-CoV-2 viral spread and innate immunity. Nature. 2020 Nov;587(7835):657-62. doi: 10.1038/s41586-020-2601-5 PMID: 32726803

36. Yan S, Wu G. Spatial and temporal roles of SARS-CoV PLpro-A snapshot. FASEB J. 2021 Jan;35(1):e21197. doi: 10.1096/fj.202002271 PMID: 33368679

37. Bekes M, van Noort GJvdH, Ekkebus R, Ovaa H, Huang TT, Lima CD. Recognition of Lys48-linked diubiquitin and deubiquitinating activities of the SARS coronavirus papain-like protease. Mol Cell. 2016 May;62(4):572-85. doi: 10.1016/j.molcel.2016.04.016 PMID: 27203180 
38. Ratia K, Kilianski A, Baez-Santos YM, Baker SC, Mesecar A. Structural basis for the ubiquitin-linkage specificity and deISGylating activity of SARS-CoV papain-like protease. PLOS Pathog. 2014 May;10(5):e1004113. doi: 10.1371/journal.ppat.1004113 PMID: 24854014

39. Durie IA, Dzimianski JV, Daczkowski CM, McGuire J, Faaberg K, Pegan SD. Structural insights into the interactions of papain-like protease 2 from the alphacoronavirus porcine epidemic diarrhea virus and ubiquitin. Acta Crystallogr D Struct Biol. 2021 Jul;77(7):943-53. doi: 10.1107/S205979832100509X PMID: 34196620

40. Clasman JR, Everett RK, Srinivasan K, Mesecar AD. Decoupling delSGylating and deubiquitinating activities of the MERS virus papain-like protease. Antiviral Res. 2020 Feb;174:104661. doi: 10.1016/i.antiviral.2019.104661 PMID: 31765674

41. Swatek KN, Aumayr M, Pruneda JN, Visser LJ, Berryman S, Kueck AF, et al. Irreversible inactivation of ISG15 by a viral leader protease enables alternative infection detection strategies. PNAS. 2018 Mar;115(10):2371-6. doi: 10.1073/pnas.1710617115 PMID: 29463763

42. Schulman BA, Harper JW. Ubiquitin-like protein activation by E1 enzymes: the apex for downstream signalling pathways. Nat Rev Mol Cell Biol. 2009 May;10(5):319-31. doi: 10.1038/nrm2673 PMID: 19352404

43. Lois LM, Lima CD. Structures of the SUMO E1 provide mechanistic insights into SUMO activation and E2 recruitment to E1. EMBO J. 2005 Feb;24(3):439-51. doi: 10.1038/sj.emboj.7600552 PMID: 15660128

44. Li FQ, Xiao H, Tam JP, Liu DX. Sumoylation of the nucleocapsid protein of severe acute respiratory syndrome coronavirus. FEBS Lett. 2005 Apr;579(11):2387-96. doi: 10.1016/i.febslet.2005.03.039 PMID: 15848177

45. Fan Z, Zhuo Y, Tan X, Zhou Z, Yuan J, Qiang B, et al. SARS-CoV nucleocapsid protein binds to hUbc9, a ubiquitin conjugating enzyme of the sumoylation system. J Med Virol. 2006 Nov;78(11):1365-73. doi: 10.1002/jmv.20707 PMID: 16998888

46. Hiscox JA. The interaction of animal cytoplasmic RNA viruses with the nucleus to facilitate replication. Virus Res. 2003 Sep;95(1):13-22. doi: 10.1016/S0168-1702(03)00160-6 PMID: 12921992

47. Hu MM, Yang Q, Xie XQ, Liao CY, Lin H, Liu TT, et al. Sumoylation promotes the stability of the DNA sensor CGAS and the adaptor STING to regulate the kinetics of response to DNA virus. Immunity. 2016 Sep;45(3):555-69. doi: 10.1016/j.immuni.2016.08.014 PMID: 27637147

48. Sun $L$, Xing $Y$, Chen $X$, Zheng $Y$, Yang $Y$, Nichols DB, et al. Coronavirus papain-like proteases negatively regulate antiviral innate immune response through disruption of STING-mediated signaling. PLOS One. 2012 Feb;7(2):e30802. doi: 10.1371/journal.pone.0030802 PMID: 22312431

49. Maringer K, Fernandez-Sesma A. Message in a bottle: lessons learned from antagonism of STING signalling during RNA virus infection. Cytokine Growth Factor Rev. 2014 Dec;25(6):669-79. doi: 10.1016/j.cytogfr.2014.08.004 PMID: 25212897

50. Nguyen LK, Dobrzynski M, Fey D, Kholodenko BN. Polyubiquitin chain assembly and organization determine the dynamics of protein activation and degradation. Front Physiol. 2014 Jan;5:4. doi: 10.3389/fphys.2014.00004 PMID: 24478717

51. Rodriquez MS, Desterro JM, Lain S, Midgley CA, Lane DP, Hay RT. SUMO-1 modification activates the transcriptional response of p53. EMBO J. 1999 Nov;18(22):6455-61. doi: 10.1093/emboj/18.22.6455 PMID: 10562557

52. Ma-Lauer Y, Carbajo-Lozoya J, Hein MY, Muller MA, Deng W, Lei J, et al. p53 down-regulates SARS coronavirus replication and is targeted by the SARS-unique domain and PLpro via E3 ubiquitin ligase RCHY1. PNAS. 2016 Aug;113(35):E5192-201. doi: 10.1073/pnas.1603435113 PMID: 27519799

53. Desterro JMP, Rodriguez MS, Hay RT. SUMO-1 modification of IKB $\alpha$ inhibits NF-kB activation. Mol Cell. 1998 Aug;2(2):233-9. doi: 10.1016/s1097-2765(00)80133-1 PMID: 9734360 
bioRxiv preprint doi: https://doi.org/10.1101/2021.10.04.462902; this version posted October 6, 2021. The copyright holder for this preprint (which was not certified by peer review) is the author/funder, who has granted bioRxiv a license to display the preprint in perpetuity. It is made available under aCC-BY 4.0 International license.

54. DeDiego ML, Nieto-Torres JL, Regla-Nava JA, Jimenez-Guardeno JM, Fernandez-Delgado R, Fett C, et al. Inhibition of NF-KB-mediated inflammation in severe acute respiratory syndrome coronavirusinfected mice increases survival. J Virol. 2014 Jan;88(2):913-24. doi: 10.1128/JVI.02576-13 PMID: 24198408

55. Banerjee S, Kumar M, Wiener R. Decrypting UFMylation: how proteins are modified with UFM1. Biomolecules. 2020 Oct;10(10):1442. doi: 10.3390/biom10101442 PMID: 33066455

56. Stephani M, Picchianti L, Gajic A, Beveridge R, Skarwan E, Hernandez VSDM, et al. A cross-kingdom conserved ER-phagy receptor maintains endoplasmic reticulum homeostasis during stress. Elife. 2020 Aug;9:e58396. doi: 10.7554/eLife.58396 PMID: 32851973

57. Wolff G, Melia CE, Snijder EJ, Barcena M. Double-membrane vesicles as platforms for viral replication. Trends in Microbiol. 2020 Jun;1839. doi: 10.1016/j.tim.2020.05.009 PMID: 32536523

58. Becker AC, Gannage M, Giese S, Hu Z, Abou-Eid S, Roubaty C, et al. Influenza A virus induces autophagosomal targeting of ribosomal proteins. Mol Cell Proteomics. 2018 Oct;17(10):1909-21. doi: 10.1074/mcp.RA117.000364 PMID: 29980615

59. Liu J, Guan D, Dong M, Yang J, Wei H, Liang Q, et al. UFMylation maintains tumor suppressor p53 stability by antagonizing its ubiquitination. Net Cell Biol. 2020 Sep;22(9):1056-63. doi: 10.1038/s41556-020-0559-z PMID: 32807901

60. Balce DR, Wang YT, McAllaster MR, Dunlap BF, Orvedahl A, Hykes BL, et al. UFMylation inhibits the proinflammatory capacity of interferon- $\gamma$-activated macrophages. PNAS. 2021 Jan;118(1):e2011763118. doi: 10.1073/pnas.2011763118 PMID: 33372156

61. Grant RA, Morales-Nebreda L, Markov NS, Swaminathan S, Querrey M, Guzman ER, et al. Circuits between infected macrophages and T cells in SARS-CoV-2 pneumonia. Nature. 2021 Feb;590(7847):635-41. doi: 10.1038/s41586-020-03148-w PMID: 33429418

62. Malakhova OA, Zhang DE. ISG15 inhibits Nedd4 ubiquitin E3 activity and enhances the innate antiviral response. J Biol Chem. 2008 Apr;283(14):8783-7. doi: 10.1074/jbc.C800030200 PMID: 18287095

63. Novelli G, Liu J, Biancolella M, Alonzi T, Novelli A, Patten JJ, et al. Inhibition of HECT E3 ligases as a potential therapy for COVID-19. Cell Death Dis. 2021 Mar;12(4):310. doi: 10.1038/s41419-02103513-1 PMID: 33762578

64. Narita T, Weinert BT, Choudhary C. Functions and mechanisms of non-histone protein acetylation. Nat Rev Mol Cell Biol. 2018 Mar;20(3):156-74. doi: 10.1038/s41580-018-0081-3 PMID: 30467427

65. Ohtake F, Saeki Y, Sakamoto K, Ohtake K, Nishikawa H, Tsuchiya H, et al. Ubiquitin acetylation inhibits polyubiquitin chain elongation. EMBO Rep. 2015 Feb;16(2):192-201. doi: 10.15252/embr.201439152 PMID: 25527407

66. Ma L, Huang C, Wang XJ, Xin DE, Wang LS, Zou QC, et al. Lysyl oxidase 3 is a dual-specificity enzyme involved in STAT3 deacetylation and deacetylimination modulation. Mol Cell. 2017 Jan;65(2):296309. doi: 10.1016/j.molcel.2016.12.002 PMID: 28065600

67. Matsuyama T, Kubli SP, Yoshinaga SK, Pfeffer K, Mak TW. An aberrant STAT pathway is central to COVID-19. Cell Death Differ. 2020 Dec;27(12):3209-25. doi: 10.1038/s41418-020-00633-7 PMID: 33037393

68. Thandapani P, O'Connor TR, Bailey TL, Richard S. Defining the RGG/RG motif. Mol Cell. 2013 Jun;50(5):613-23. doi: 10.1016/j.molcel.2013.05.021 PMID: 23746349

69. Nabeel-Shah S, Lee H, Ahmed N, Marcon E, Farhangmehr S, Pu S, et al. SARS-CoV-2 nucleocapsid protein attenuates stress granule formation and alters gene expression via direct interaction with host mRNAs. bioRxiv [Preprint]. 2020 Oct. doi: 10.1101/2020.10.23.342113

70. Cai T, Yu Z, Wang Z, Liang C, Richard S. Arginine methylation of SARS-CoV-2 nucleocapsid protein regulated RNA binding, its ability to suppress stress granule formation, and viral replication. J Biol Chem. 2021 Jul;297(1):100821. doi: 10.1016/j.jbc.2021.100821 PMID: 34029587 
71. Nemeroff ME, Barabino SM, Li Y, Keller W, Krug RM. Influenza virus NS1 protein interacts with the cellular 30 kDa subunit of CPSF and inhibits 3' end formation of cellular pre-RNAs. Mol Cell. 1998 Jun;1(7):991-1000. doi: 10.1016/s1097-2765(00)80099-4 PMID: 9651582

72. Meola N, Domanski M, Karadoulama E, Chen Y, Gentil C, Pultz D, et al. Identification of a nuclear exosome decay pathway for processed transcripts. Mol Cell. 2016 Nov;64(3):520-33. doi: 10.1016/j.molcel.2016.09.025 PMID: 27871484

73. Wu HY, Ke TY, Liao WY, Chang NY. Regulation of coronaviral poly(A) tail length during infection. PLOS One. $2013 \mathrm{Jul}$;8(7):e70548. doi: 10.1371/journal.pone.0070548 PMID: 23923003

74. Peng YH, Lin CH, Lin CN, Lo CY, Tsai TL, Wu HY. Characterization of the role of hexamer AGUAAA and poly(A) tail in coronavirus polyadenylation. PLOS One. 2016 Oct;11(10):e0165077. doi: 10.1371/journal.pone.0165077 PMID: 27760233

75. Kim D, Lee YS, Jung SJ, Yeo J, Seo JJ, Lee YY, et al. Viral hijacking of the TENT4-ZCCHC14 complex protects viral RNAs via mixed tailing. Nat Struct Mol Biol. 2020 Jun;27(6):581-8. doi: 10.1038/s41594-020-0427-3 PMID: 32451488

76. Miller JE, Reese JC. Ccr4-Not complex: the control freak of eukaryotic cells. Crit Rev Biochem Mol Biol. 2012 Jul:47(4):315-33. doi: 10.3109/10409238.2012.667214 PMID: 22416820

77. Daffis S, Szretter KJ, Schriewer J, Li J, Youn S, Errett J, et al. 2'-O methylation of the viral mRNA cap evades host restriction by IFIT family members. Nature. 2010 Nov;468(7322):452-6. doi: 10.1038/nature09489 PMID: 21085181

78. Viswanathan T, Arya S, Chan SH, Qi S, Dai N, Misra A, et al. Structural basis of RNA cap modification by SARS-CoV-2. Nat Commun. 2020 Jul;11:3718. doi: 10.1038/s41467-020-17496-8 PMID: 32709886

79. Kincaid RP, Lam VL, Chirayil RP, Randall G, Sullivan CS. RNA triphosphatase DUSP11 enables exonuclease XRN-mediated restriction of hepatitis C virus. PNAS. 2018 Aug;115(32):8197-202. doi: 10.1073/pnas.1802326115 PMID: 30038017

80. Choi JH, Burke JM, Szymanik KH, Nepal U, Battenhouse A, Lau JT, et al. DUSP11-mediated control of 5'-triphosphate RNA regulates RIG-I sensitivity. Genes Dev. 2020 Dec;34(23-24):1697-712. doi: 10.1101/gad.340604.120 PMID: 33184222

81. Choi JH, Sullivan CS. DUSP11 and triphosphate RNA balance during virus infection. PLOS Pathog. 2021 Jan;17(1):e1009145. doi: 10.1371/journal.ppat.1009145 PMID: 33444401

82. Bonaventure B, Rebendenne A, de Gracia FG, McKellar J, Gracias S, Labaronne E, et al. A genomewide CRISPR/Cas9 knock-out screen identifies DEAD box RNA helicase DDX42 as a broad antiviral inhibitor. bioRxiv [Preprint]. 2020 Oct. doi: 10.1101/2020.10.28.359356

83. Temerozo J, Fintelman-Rodrigues N, Santos MC, Hottz E, Sacramento C, et al. Human endogenous retrovirus $\mathrm{K}$ activation in the lower respiratory tract of severe COVID-19 patients associated with early mortality. Research Square [Preprint]. 2021 May. doi: 10.21203/rs.3.rs-514541/v1

84. Tyagi R, Li W, Parades D, Bianchet MA, Nath A. Inhibition of human endogenous retrovirus-K by antiretroviral drugs. Retrovirology. 2017 Mar;14:21. doi: 10.1186/s12977-017-0347-4 PMID: 28330477

85. Mahdi M, Motyan JA, Szojka ZI, Golda M, Miczi M, Tozser J. Analysis of the efficacy of HIV protease inhibitors against SARS-CoV-2's main protease. Virol J. 2020 Nov;17(1):190. doi: 10.1186/s12985020-01457-0 PMID: 33243253

86. Cao B, Wang Y, Wen D, Liu W, Wang J, Fan G, et al. A trial of lopinavir-ritonavir in adults hospitalized with severe COVID-19. N Engl J Med. 2020 May;382(19):1787-99. doi: 10.1056/NEJMoa2001282 PMID: 32187464 